\title{
Near-Ambient Pressure XPS and NEXAFS Study of a Superbasic lonic Liquid with $\mathrm{CO}_{2}$
}

\section{Supporting Information}

Jordan Cole ${ }^{*}$, , Zoë Henderson ${ }^{\dagger}, \#$, Andrew G. Thomas ${ }^{\ddagger}$, Claudia L. Compeán-González ${ }^{\ddagger}$, Adam

J. Greerll, Christopher Hardacrell, Federica Venturini ${ }^{\S}$, Wilson Quevedo Garzon ${ }^{\S, \perp}$, Pilar

Ferrer $^{\S}$, David C. Grinter ${ }^{\S}$, Georg Held ${ }^{\S}$, and Karen L. Syres ${ }^{*},+$

$\dagger \quad$ Jeremiah Horrocks Institute for Mathematics, Physics and Astronomy, University of

Central Lancashire, Preston, PR1 2HE, UK

‡ Department of Materials Science, Photon Science Institute and Henry Royce Institute, University of Manchester, Manchester, M13 9PL, UK

Department of Chemical Engineering and Analytical Science, University of

Manchester, Manchester, M13 9PL, UK

$\S \quad$ Diamond Light Source, Didcot, OX11 ODE, UK

*Email: KSyres@uclan.ac.uk; JCole4@uclan.ac.uk

\section{XPS Spectra}

Figure S1 shows the survey spectra of a thin film of $\left[\mathrm{P}_{66614}\right][$ benzim] as presented, while exposed to $10^{-6}$ mbar $\mathrm{CO}_{2}, 1$ mbar $\mathrm{CO}_{2}, 5 \mathrm{mbar} \mathrm{CO}_{2}$, and after exposure. A survey scan of the clean $\mathrm{TiO}_{2}(110)$ crystal was taken prior to deposition of the IL. These were all taken with a photon energy of $1200 \mathrm{eV}$. The assignments and binding energies (BEs) of fitted components across the $C 1 s, N$ s, $O$ 1s and $P 2 p$ regions is summarised in Table $S 1$.

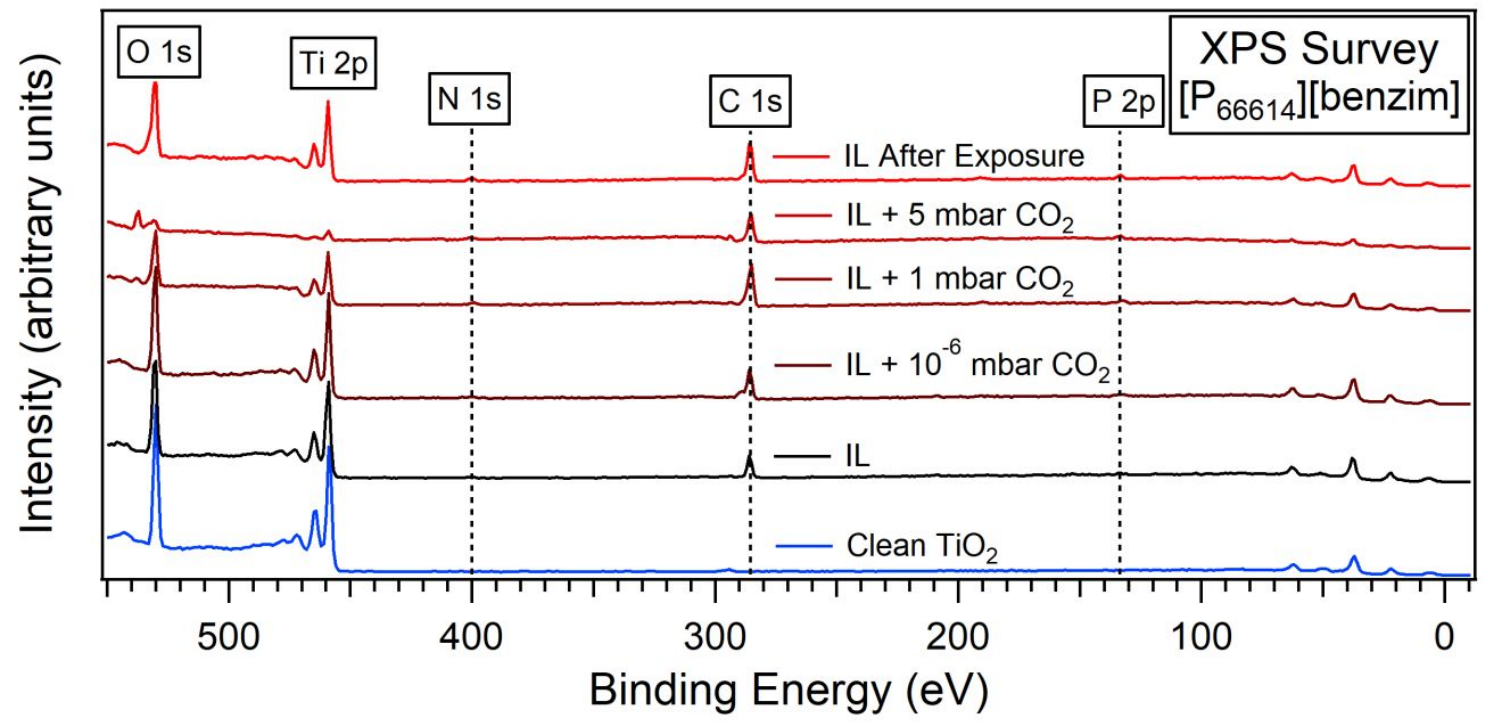

Figure S1. XPS survey spectra of $\left[P_{66614}\right]\left[\right.$ benzim] subject to various exposures of $\mathrm{CO}_{2}$. Highlighted are the peaks associated with atoms in the $\mathrm{TiO}_{2}(110)$ crystal substrate (O 1s, Ti 2p) and the IL (N 1s, C 1s, P 2p). 
Narrow scans of the Ti $2 p$ region for the $\mathrm{IL}\left[\mathrm{P}_{66614}\right][$ benzim] are shown in Figure $\mathrm{S} 2$ before exposure to $\mathrm{CO}_{2}$; upon exposure to $10^{-6} \mathrm{mbar}, 1 \mathrm{mbar}$ and $5 \mathrm{mbar}$ of $\mathrm{CO}_{2}$; and after exposure. The region consists of two peaks at 458.7 and $464.5 \mathrm{eV}$ which occur due to spin-orbit coupling, assigned as $2 p_{3 / 2}$ and $2 p_{1 / 2}$, respectively. The spectra show very little change through the exposure stages, except for increasing noise with higher pressures of $\mathrm{CO}_{2}$ due to increased attenuation of photoelectrons.

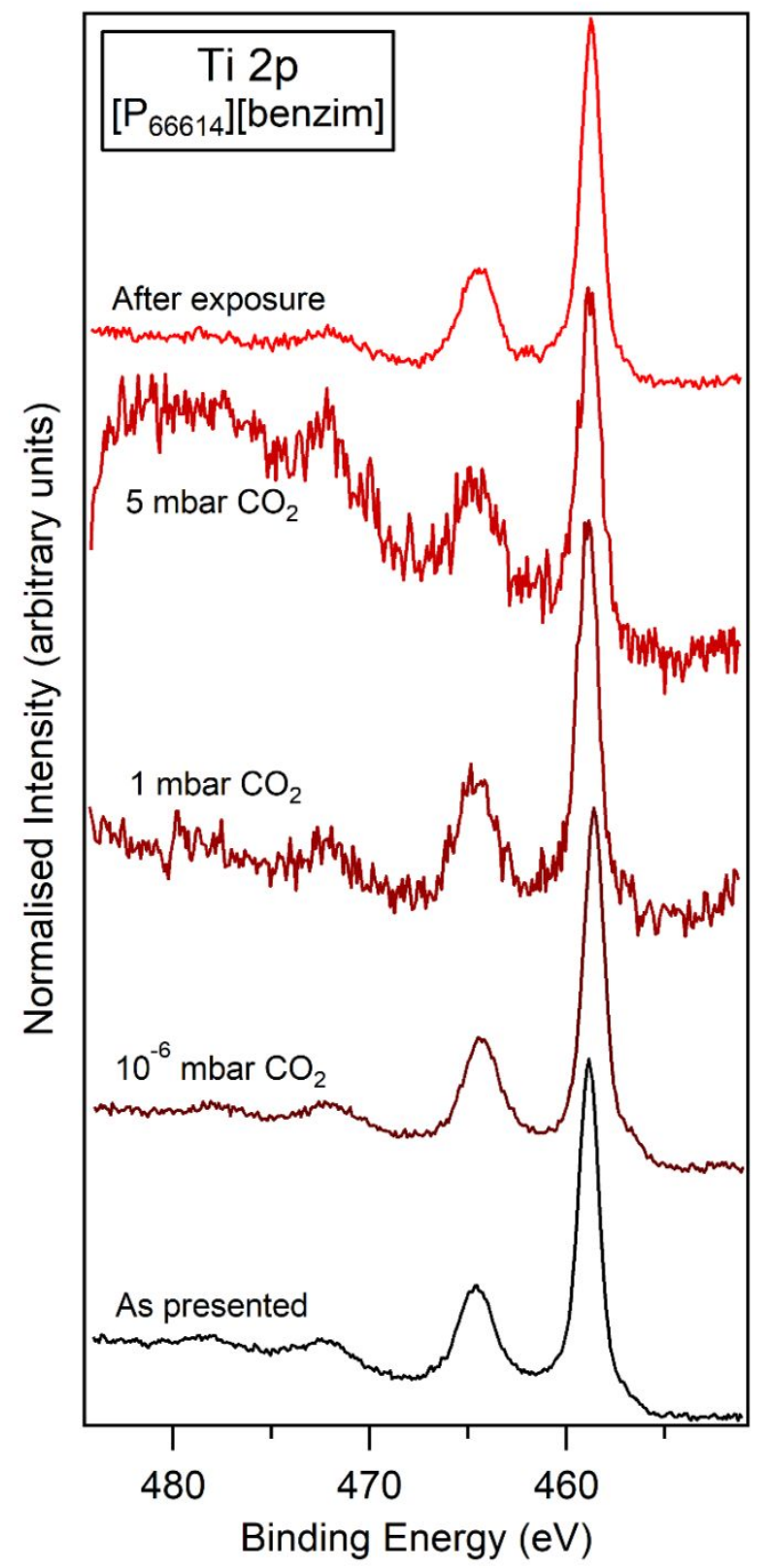

Figure S2. Ti $2 p$ region for multilayer $\left[\mathrm{P}_{66614}\right]\left[\right.$ benzim] as presented, exposed to $10^{-6} \mathrm{mbar} \mathrm{CO}_{2}, 1 \mathrm{mbar} \mathrm{CO}_{2}, 5 \mathrm{mbar} \mathrm{CO}_{2}$, and after exposure (bottom to top, respectively).

O 1s spectra taken over the same exposure stages are shown in Figure S3. When the IL is exposed to higher pressures of $\mathrm{CO}_{2}$ ( 1 mbar and 5 mbar), the spectra can be fitted with four components at binding energies of 530.0, 531.1, 532.2 and $533.3 \mathrm{eV}$. Note that the intense gas-phase $\mathrm{CO}_{2}$ peak at $537.2 \mathrm{eV}$ is not shown in Figure $\mathrm{S} 3$ in order to make the region clearer. The component at $530.0 \mathrm{eV}$ is attributed to $\mathrm{O}$ atoms in the $\mathrm{TiO}_{2}$ lattice and is detectable 
because the IL film is sufficiently thin (approximately $5 \mathrm{~nm}$ ). ${ }^{1}$ We suspect there is a mixture of protonated and unprotonated carbamate species in the sample, therefore, the component at $531.1 \mathrm{eV}$ is attributed to unprotonated carbamate, while the remaining components at 532.2 and $533.3 \mathrm{eV}$ are attributed to $\mathrm{C}=\mathrm{O}$ and $\mathrm{C}-\mathrm{OH}$ in the protonated carbamate group, respectively. ${ }^{2-4}$ Protonation of the anion is likely to arise due to contamination of water vapour. The $\mathrm{TiO}_{2}$ lattice $\mathrm{O}$ component at $530.0 \mathrm{eV}$ reduces in intensity with increasing $\mathrm{CO}_{2}$ pressure. Upon exposure to the highest pressure of 5 mbar $\mathrm{CO}_{2}$, the $\mathrm{C}=\mathrm{O}$ and $\mathrm{C}-\mathrm{OH}$ components contribute to a pronounced protonated carbamate feature. After removal of $\mathrm{CO}_{2}$, the protonated carbamate components are still present (top spectrum of Figure S3), however, in the IL as presented and $\mathrm{IL}+10^{-6}$ mbar $\mathrm{CO}_{2}$ stages the $\mathrm{C}-\mathrm{OH}$ component is too small to be fitted.

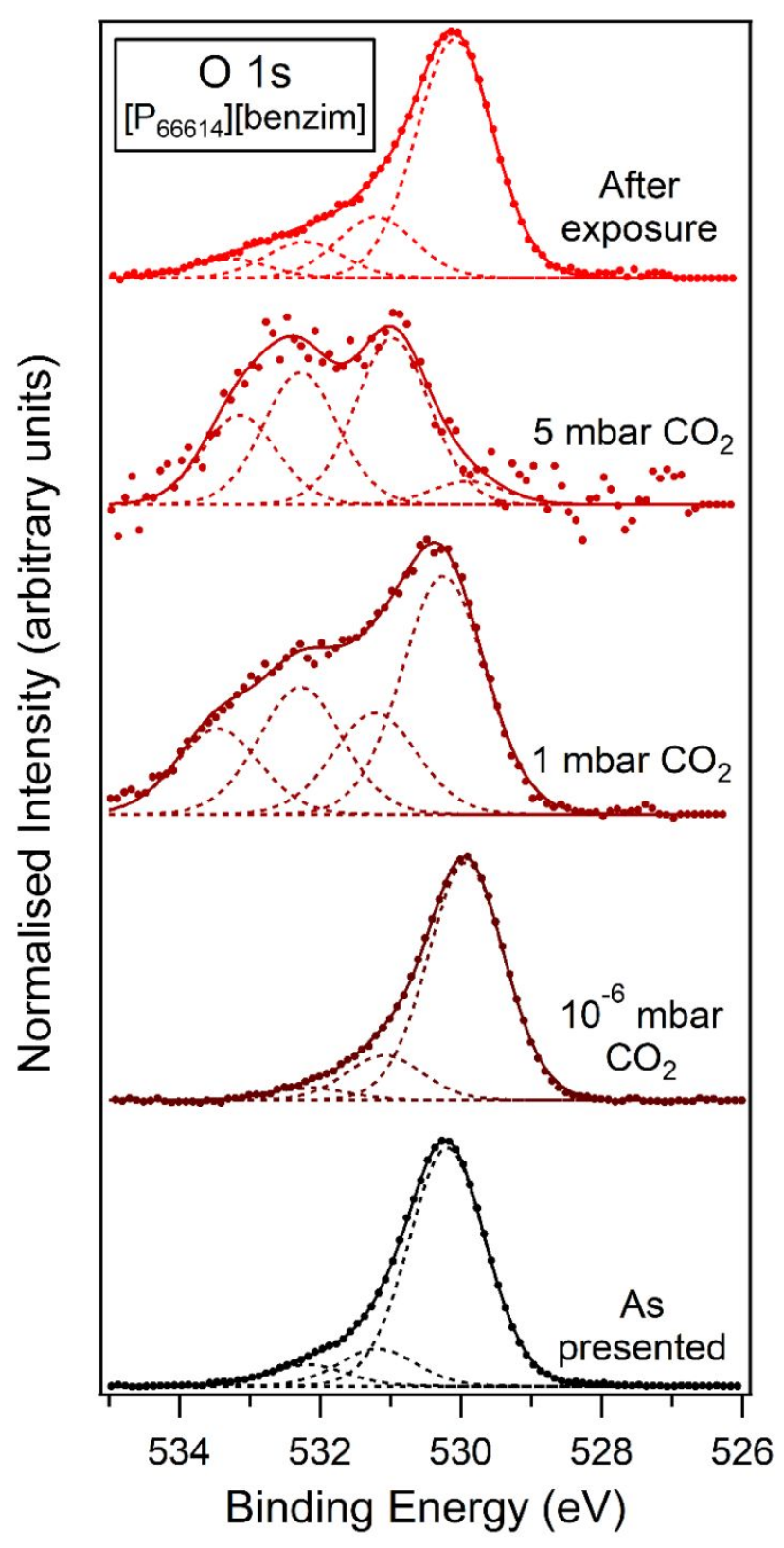


Figure S3. $O$ 1s region recorded at a photon energy of $830 \mathrm{eV}$ for multilayer [ $\left.P_{66614}\right]$ [benzim] as presented, while exposed to various pressures of $\mathrm{CO} 2$, and after exposure.

The $\mathrm{O} 1 \mathrm{~s}$ spectra in Figure $\mathrm{S} 3$ have been normalised to the intensity of the $\mathrm{TiO}_{2}$ lattice $\mathrm{O}$ peak at $530.0 \mathrm{eV}$. However, there is a dramatic relative decrease in the $530.0 \mathrm{eV}$ component of the $\mathrm{IL}+5$ mbar $\mathrm{CO}_{2}$ spectrum as the $\mathrm{IL}$ absorbs more $\mathrm{CO}_{2}$. Therefore, this spectrum was normalised such that the intensities of high BE components at 532.2 and $533.3 \mathrm{eV}$ have the same intensities as those for $1 \mathrm{mbar} \mathrm{CO}_{2}$.

\begin{tabular}{|c|c|c|}
\hline Region & Binding Energy $(\mathrm{eV}) \pm 0.1 \mathrm{eV}$ & Assignment \\
\hline C 1s & $\begin{array}{l}285.0 \\
285.9 \\
286.8 \rightarrow 287.1 \\
289.0^{a} \\
293.3\end{array}$ & $\begin{array}{l}\mathrm{C}_{\text {aliphatic }} \\
\mathrm{C}_{\text {hetero }} \\
\mathrm{C}_{\text {anion }} \\
\text { Carbamate } \\
\text { Gas-Phase } \mathrm{CO}_{2}\end{array}$ \\
\hline N 1s & $\begin{array}{l}398.6 \\
400.0 \\
401.1\end{array}$ & $\begin{array}{l}N^{1,2} \\
N^{2, r} \\
N^{1, r}\end{array}$ \\
\hline 0 1s & $\begin{array}{l}530.0^{a} \\
531.1 \\
532.2 \\
533.3^{a} \\
537.2 \\
\end{array}$ & $\begin{array}{l}\mathrm{TiO}_{2} \text { lattice } \mathrm{O} \\
\text { Carbamate } \\
\mathrm{C}=\mathrm{O}^{b} \\
\mathrm{C}-\mathrm{OH}^{b} \\
\text { Gas-Phase } \mathrm{CO}_{2}\end{array}$ \\
\hline$P 2 p$ & $\begin{array}{l}130.4 \\
131.3 \\
132.4 \rightarrow 132.8 \\
133.3 \rightarrow 133.7 \\
135.6 \\
136.5\end{array}$ & $\begin{array}{l}\text { THP } 2 \mathrm{p}_{3 / 2} \\
\text { THP } 2 \mathrm{p}_{1 / 2} \\
{\left[\mathrm{P}_{66614}\right]^{+} 2 \mathrm{p}_{3 / 2}} \\
{\left[\mathrm{P}_{66614}\right]^{+} 2 \mathrm{p}_{1 / 2}} \\
\text { Phosphine oxide } 2 \mathrm{p}_{3 / 2} \\
\text { Phosphine oxide } 2 \mathrm{p}_{1 / 2}\end{array}$ \\
\hline
\end{tabular}

Table S1. Assignments and BEs of fitted components in the $C 1 s, N 1 s, O$ s and $P 2 p$ regions for [ $P_{66614}$ [benzim] before exposure, while exposed to $\mathrm{CO}_{2}$ at various pressures, and after exposure. These were taken at photon energies of 585, 700, 830 , and $430 \mathrm{eV}$, respectively, for each of the above regions. The arrow $(\rightarrow)$ denotes a chemical shift due to the absorption of $\mathrm{CO}_{2} \cdot{ }^{a} \mathrm{BES}$ differ by $\pm 0.2 \mathrm{eV} .{ }^{b}$ Within the protonated carbamate group.

\section{Grazing Emission XPS Study}

XPS spectra of [ $\left.\mathrm{P}_{66614}\right]$ [benzim] were taken at grazing emission ( $\mathrm{GE}, 40^{\circ}$ from normal emission) over the $C$ 1s region as shown in Figure $S 4$, providing more surface-sensitive measurements. There is little change in the overall shape of the $C 1$ s peak when taken at GE compared to normal emission (NE) except for a slight reduction in the intensity of the $C_{\text {hetero }}$ component at $285.9 \mathrm{eV}$. From this we can deduce that the concentration of $\mathrm{C}_{\mathrm{aliphatic}}, \mathrm{C}_{\text {hetero }}$ and $\mathrm{C}_{\text {anion }}$ species 
does not vary significantly throughout the sample depth. The peaks at 293 and $296 \mathrm{eV}$ are assigned as $K 2 p_{3 / 2}$ and $K 2 p_{1 / 2}$, respectively. They become more pronounced for $G E$, indicating surface contamination with $\mathrm{K}$. Shake-up satellites may also manifest at similar BEs but the presence of a feature at approximately $295 \mathrm{eV}$ in the clean $\mathrm{TiO}_{2}$ survey spectrum would suggest this is $\mathrm{K}$ contamination.
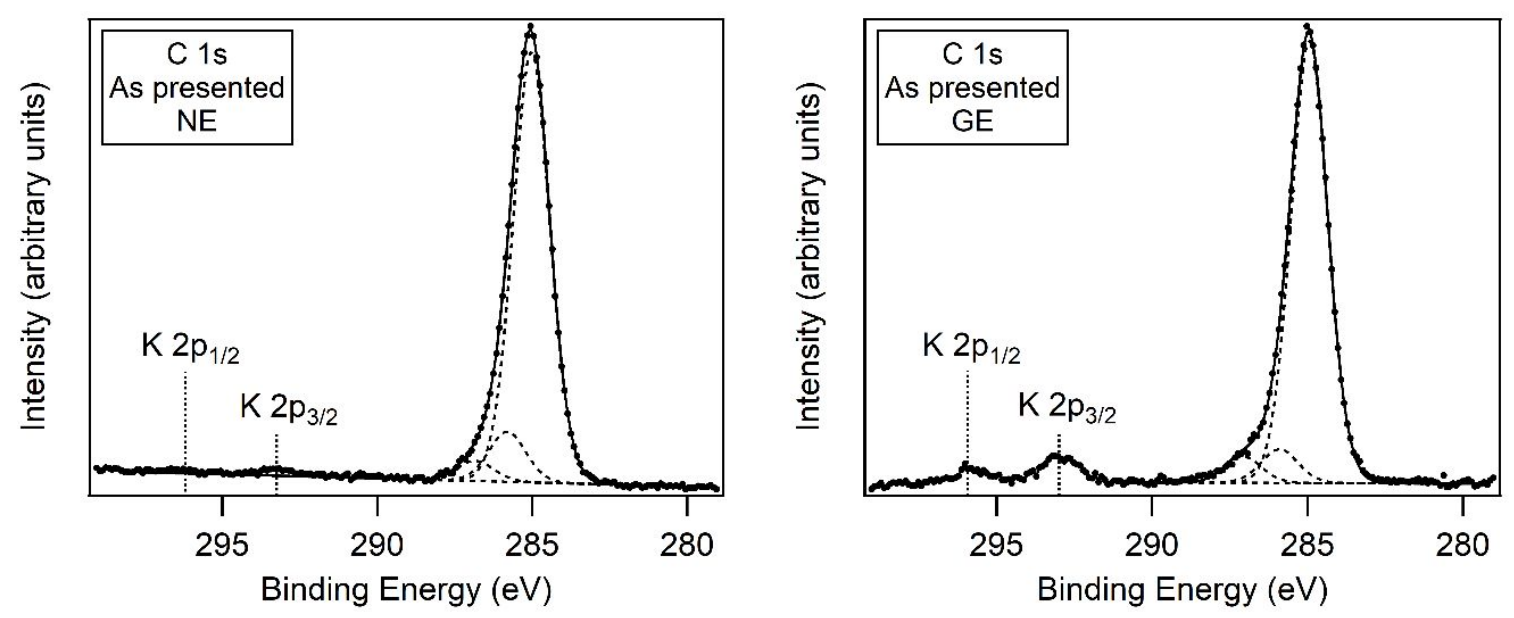

Figure S4. C 1s normal emission (NE) and grazing emission (GE) XPS spectra of [ $\left.P_{66614}\right]$ [benzim].

GE measurements over the $\mathrm{N} 1 \mathrm{~s}$ region were taken before exposure to $\mathrm{CO}_{2}$ and compared to those taken at NE as shown in Figure S5. This provides an insight into how the concentration of the $\mathrm{N}^{1, \mathrm{r}}(401.1 \mathrm{eV})$ and $\mathrm{N}^{2, \mathrm{r}}(400.0 \mathrm{eV})$ reaction species changes with depth into the sample. Compared to NE, the $\mathrm{N} 1 \mathrm{~s}$ peak taken at GE in Figure $\mathrm{S} 5$ has a more intense $\mathrm{N}^{1,2}(398.6 \mathrm{eV})$ component relative to the two reaction-based components. This suggests that there is a greater concentration of reacted anions in the deeper layers of the thin film.

As discussed by Henderson et al., the anion can also react with ambient water vapour, resulting in components at similar BEs of 397.5, 398.5 and $400.0 \mathrm{eV} .{ }^{5}$ Our results in Figure 1 (c) show a significant increase in BE for these components for multilayer versus bulk deposition. Wagstaffe et al. reported a dependence of peak position on overlayer thickness, finding that components undergo a downward shift in BE when deposited as a thin film compared to bulk deposition. ${ }^{6}$ Coverage-dependent chemical shifts have been found for thin films of the IL $\left[\mathrm{C}_{1} \mathrm{C}_{1} \mathrm{Im}\right]\left[\mathrm{Tf}_{2} \mathrm{~N}\right]$ deposited on Au (111) via physical vapour deposition by Cremer et al. ${ }^{7}$ They reported a similar dependence to Wagstaffe et al. with the addition that the peak positions for thin films of increasing thicknesses did not tend to those of a bulk film but exceeded them by as much as $0.2 \mathrm{eV}$ ( 4.5 monolayer film). This may explain the higher BEs reported here for our thin film in Figure 1(c) compared to previous reports for bulk thicknesses. 


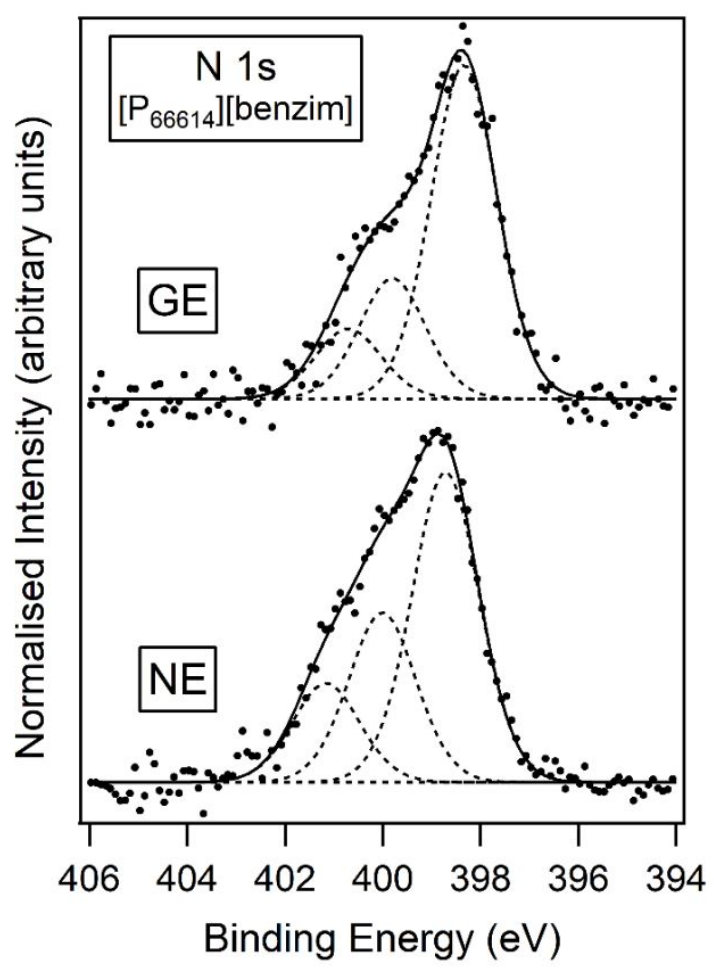

Figure S5. $N$ 1s spectra of multilayer [ $\left.P_{66614}\right]$ [benzim] (as presented) recorded at a photon energy of $700 \mathrm{eV}$ at normal and grazing emissions.

GE measurements over the $P 2 p$ region (Figure $\mathrm{S} 6$ ) show a more intense $133 \mathrm{eV}\left[\mathrm{P}_{66614}\right]^{+}$peak at $\mathrm{GE}$ compared to $\mathrm{NE}$ before exposure to $\mathrm{CO}_{2}$, suggesting there is a greater concentration of $\left[\mathrm{P}_{66614}\right]^{+}$cations at the surface compared to the bulk.

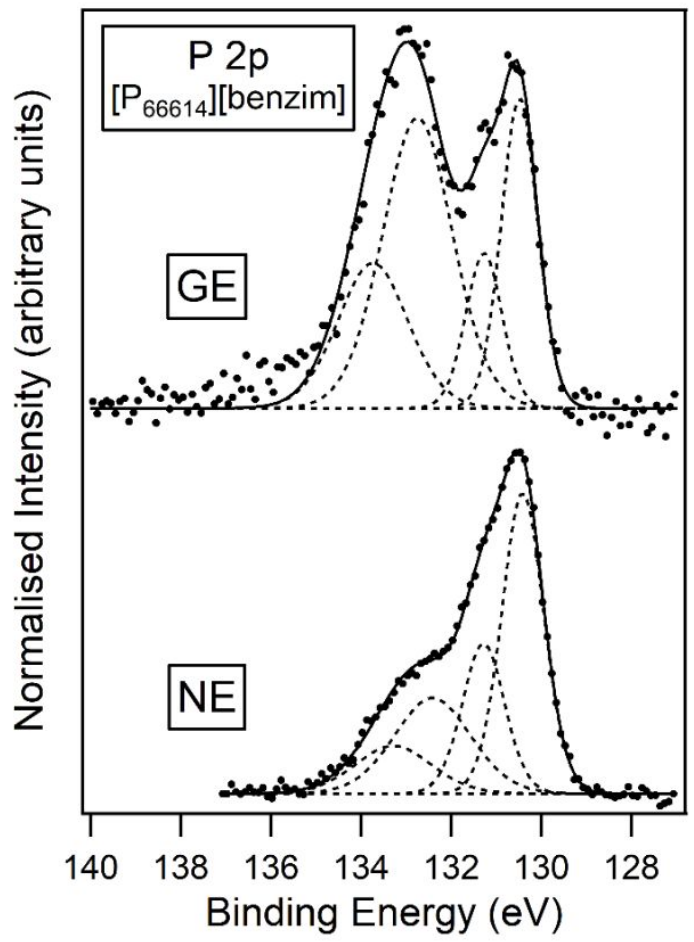

Figure S6. $P 2 p$ spectra of multilayer $\left[P_{66614}\right][$ benzim] (as presented) recorded at a photon energy of $430 \mathrm{eV}$ at normal and grazing emissions. 
GE measurements were also taken over the $\mathrm{O}$ 1s region and compared to NE measurements of the IL thin film and a clean $\mathrm{TiO}_{2}(110)$ substrate, shown in Figure S7. There is no change in the spectra of the IL thin film when taken at GE compared to NE.

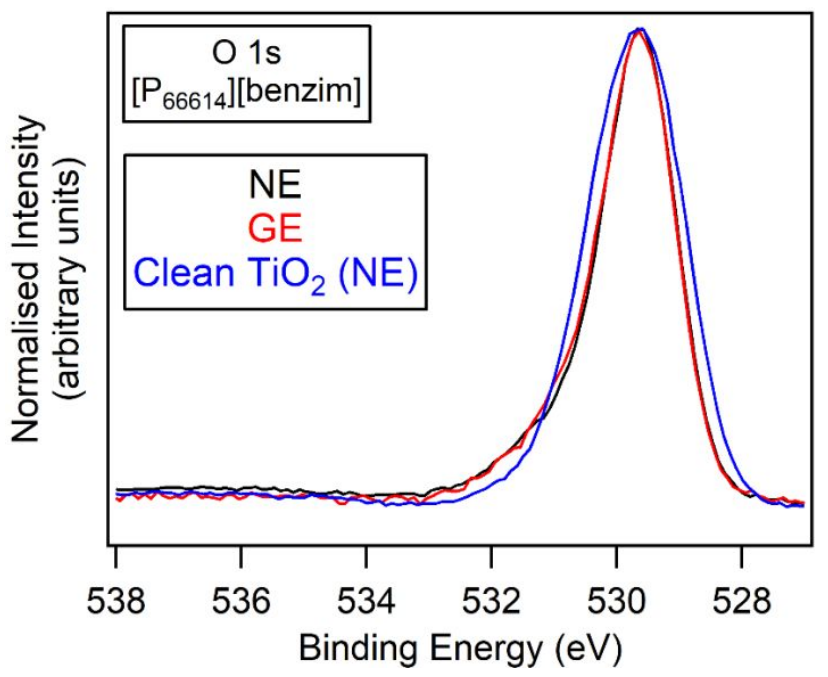

Figure S7. $O$ 1s normal and grazing emission of [ $\left.P_{66614}\right]\left[\right.$ benzim]. This is compared to clean $\mathrm{TiO}_{2}(110)$ at normal emission.

\section{$\underline{\text { NEXAFS CO}}{ }_{2}$ Gas Correction}

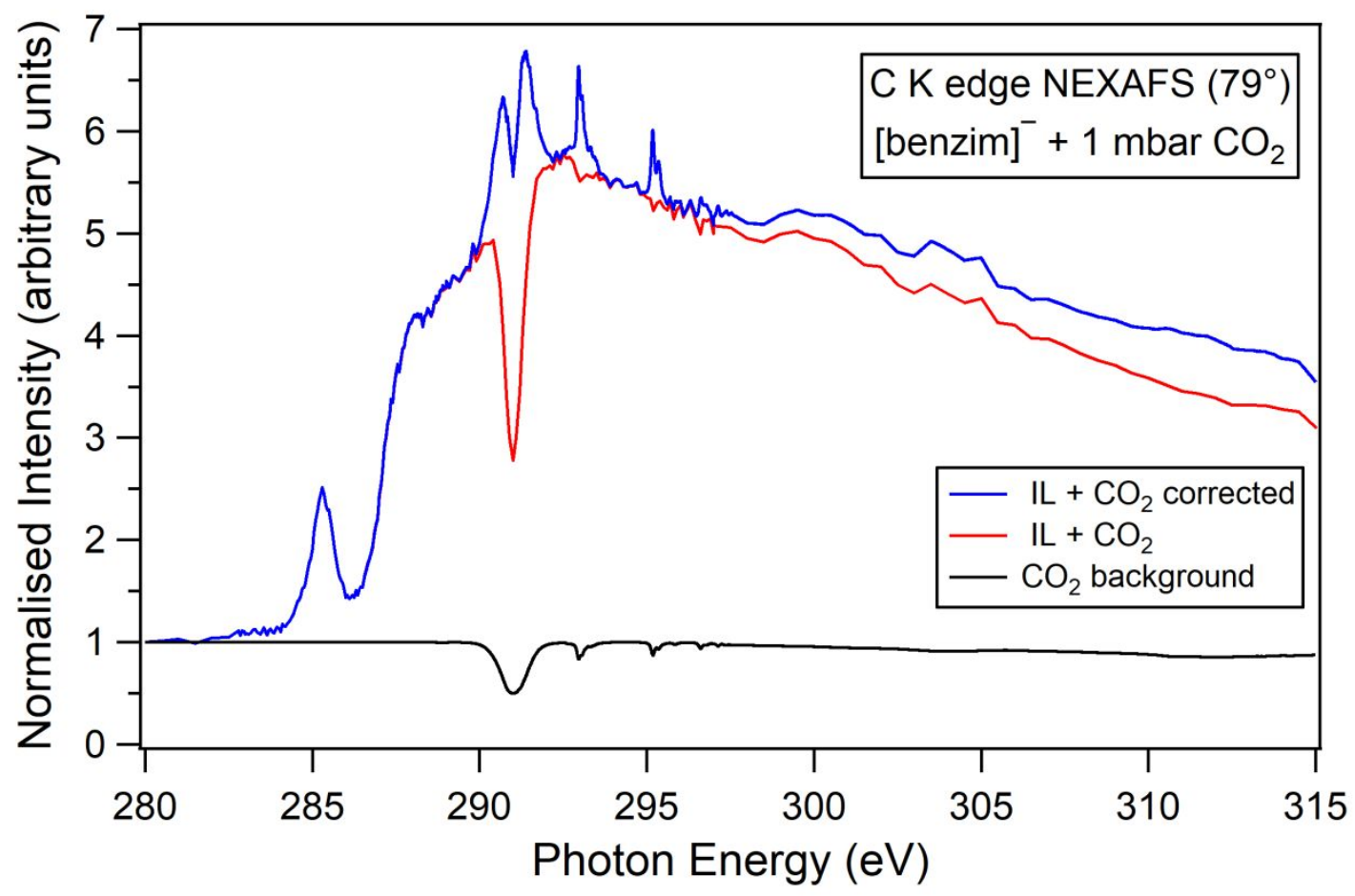

Figure S8. $C K$ edge NEXAFS of $\left[P_{66614}\right]\left[\right.$ benzim] exposed to $1 \mathrm{mbar} \mathrm{CO}_{2}$. The experimental data (red) was divided by the background gas-phase $\mathrm{CO}_{2}$ signal (black), resulting in a $\mathrm{CO}_{2}$-corrected spectrum (blue).

The $\mathrm{CO}_{2}$ gas in the NAP cell absorbs incoming X-rays at certain energies, introducing dips in the $\mathrm{CK}$ edge NEXAFS spectrum for $\left[\mathrm{P}_{66614}\right]$ [benzim] exposed to $1 \mathrm{mbar} \mathrm{CO}_{2}$ (red line) in Figure S8. This was divided by the background signal due to gas-phase $\mathrm{CO}_{2}$ (black line), which 
removes the large dip at $291 \mathrm{eV}$ but has the adverse effect of introducing small spikes into the background-corrected spectrum (blue line).

In NEXAFS measurements of the IL, we also corrected for the $\mathrm{TiO}_{2}$ substrate onto which the IL is deposited. This was done by dividing the scan of the IL by the scan of the clean $\mathrm{TiO}_{2}$ substrate. The example given in Figure $\mathrm{S} 9$ shows $\mathrm{N} \mathrm{K}$ edge measurements of the IL exposed to 1 mbar of $\mathrm{CO}_{2}$ (gold line), the clean $\mathrm{TiO}_{2}$ signal (black), and the background-corrected IL + $\mathrm{CO}_{2}$ signal obtained through division of the $\mathrm{TiO}_{2}$ spectrum.

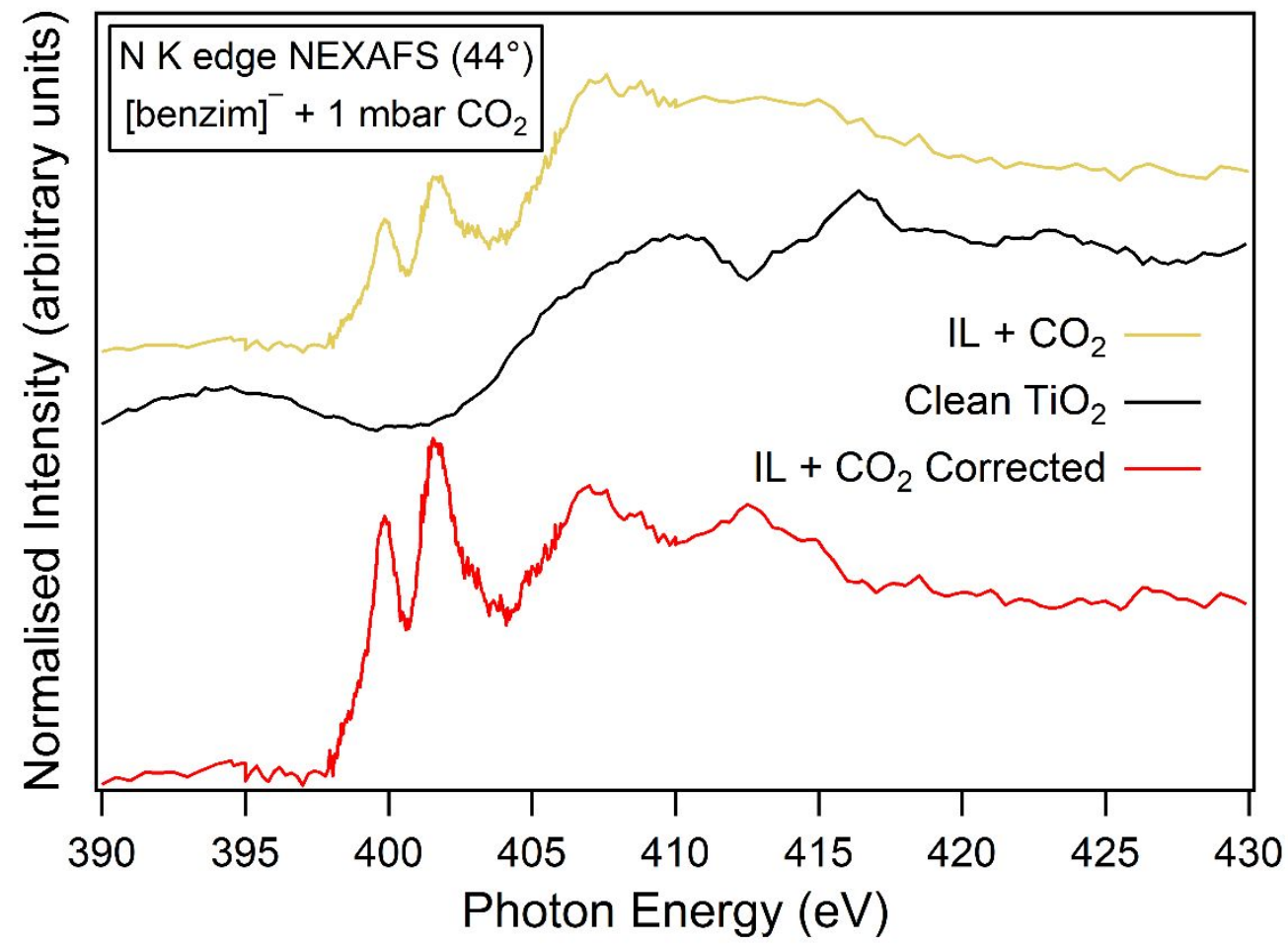

Figure S9: $N \mathrm{~K}$ edge NEXAFS of [ $\left.\mathrm{P}_{66614}\right]$ [benzim] exposed to $1 \mathrm{mbar} \mathrm{CO}_{2}$ with a removed $\mathrm{TiO}_{2}$ background signal. The experimental data (gold) was divided by the clean $\mathrm{TiO}_{2}$ substrate signal (black), resulting in a backgroundcorrected IL $+\mathrm{CO}_{2}$ spectrum (red).

\section{Experimental N K edge NEXAFS}

NEXAFS can be used to determine the orientation of [benzim] ${ }^{-}$anions in the IL thin film by comparing changes in the relative intensities of the $\pi^{*}$ and $\sigma^{*}$ peaks at different X-ray angles of incidence. $\pi^{*}$ and $\sigma^{*}$ resonances are maximised when the electric field vector of the polarised synchrotron light is parallel to the spatial orientation of the final state molecular orbital, i.e. resonances are maximum when the incident $\mathrm{X}$-rays are perpendicular to the molecular orbital. ${ }^{8}$

$\mathrm{N} \mathrm{K}$ edge NEXAFS of the IL as presented in Figure S10 has a feature at approximately $400 \mathrm{eV}$ consisting of two peaks at photon energies of 399.9 and $401.7 \mathrm{eV}$. These peaks are attributed to $N$ is $\rightarrow \pi^{*}$ transitions. There is a broader peak at $408.0 \mathrm{eV}$ attributed to $\mathrm{N}$ is $\rightarrow \sigma^{*}$ transitions. There is no definitive evidence of realignment of [benzim] anions in the as presented film in Figure S10 due to the noisy nature of the spectra. Walsh et al. reported a split $\pi^{*}$ peak in multilayer benzimidazole on $\mathrm{Cu}(100)$ at similar photon energies, and also for 
the similarly-structured benzotriazole on $\mathrm{Cu}(100) .{ }^{9}$ Grillo et al. reported a split $\pi^{*}$ peak at different positions for benzotriazole on $\mathrm{Au}(111)$, revealing that the positions of these split $\pi^{*}$ resonances vary depending on the substrate onto which the molecules are deposited. ${ }^{10}$ Splitting of the $\pi^{*}$ peak is due to initial state effects, arising from chemical shifts between different environments of $\mathrm{N}$ atoms in the imidazolide ring. ${ }^{1,11}$ The ions in these examples above are not charged and the $\mathrm{N}$ atoms would, therefore, be inequivalent, resulting in a split $\pi^{*}$ peak. However, our DFT simulations of the isolated [benzim] ${ }^{-}$anion (see Figure S14) show that both $\mathrm{N}$ atoms contribute to the split $\pi^{*}$ peak equally, implying they share the same chemical environment. The origin of the split $\pi^{*}$ peak for [benzim] ${ }^{-}$will be discussed below.

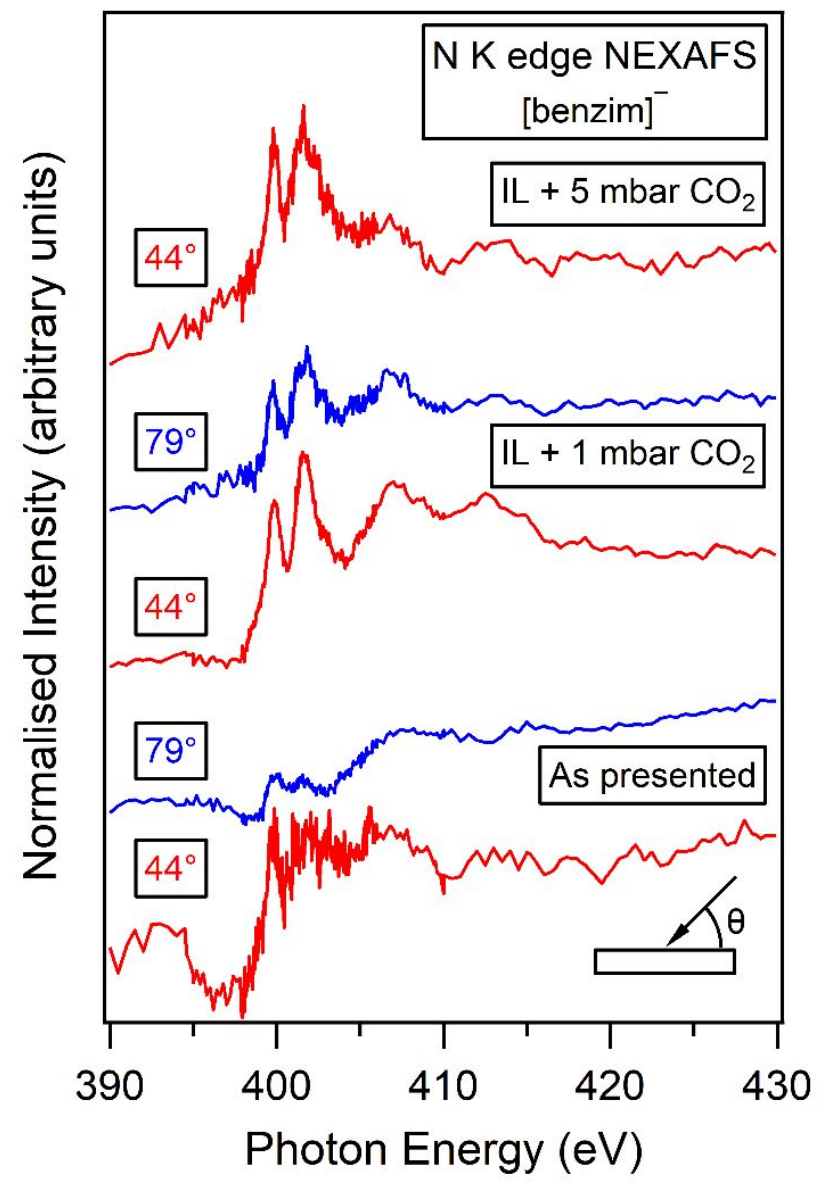

Figure S10. N K edge NEXAFS spectra of [ $\left.P_{66614}\right]\left[\right.$ benzim] as presented (bottom), when exposed to $1 \mathrm{mbar}$ of $\mathrm{CO}_{2}$ (middle) and when exposed to 5 mbar of $\mathrm{CO}_{2}$ (top), for the $X$-ray beam incident at angles of $44^{\circ}$ and $79^{\circ}$ from the IL surface.

To investigate the realignment of [benzim]' anions upon absorption of $\mathrm{CO}_{2}$ the IL was subject to two pressures of $\mathrm{CO}_{2}, 1 \mathrm{mbar}$ and $5 \mathrm{mbar}$, as shown in Figure S10. Both the $\pi^{*}$ and $\sigma^{*}$ features become more prominent than those for the IL as presented, particularly the split $\pi^{*}$ peak. However, there is no obvious change in the relative intensity of these $\pi^{*}$ and $\sigma^{*}$ peaks due to the noisy nature of the data. As found with $1 \mathrm{mbar} \mathrm{CO}_{2}$, when the $\mathrm{IL}$ is exposed to the higher 5 mbar $\mathrm{CO}_{2}$ pressure we still see a split $\pi^{*}$ peak. However, the higher-energy $\pi^{*}$ peak at $401.7 \mathrm{eV}$ is less intense relative to the lower-energy peak at $399.9 \mathrm{eV}$ compared to $1 \mathrm{mbar}$ $\mathrm{CO}_{2}$. The lower-energy peak remains unchanged with $\mathrm{CO}_{2}$ pressure, while all other features reduce in relative intensity with increased $\mathrm{CO}_{2}$ pressure. At a 5 mbar pressure the $\pi^{*}$ peaks 
are much more intense relative to the $\sigma^{*}$ peak, suggesting that the ring of the anion orients more in-plane than it does when exposed to a lower pressure of $1 \mathrm{mbar}$ (i.e. the anion ring lies closer to the surface with higher $\mathrm{CO}_{2}$ pressures). We do not have measurements at another angle to verify this, however, from results in Figure S15 that will be discussed later, we find that $\mathrm{N}^{1}$ provides the greatest contribution to the $\sigma^{*}$ peak. This is the site at which carbamate forms, and we would therefore expect a higher intensity $\sigma^{*}$ peak upon exposure to higher pressures of $\mathrm{CO}_{2}$ (5 mbar).

\section{NEXAFS DFT Simulations}

Density Functional Theory (DFT) simulations were carried out in the software StoBe-deMon, prior to which the isolated anion of the IL was geometry optimised in Avogadro. These simulations are not angle-resolved, meaning they are representative of anions being randomly oriented with respect to the incident X-ray beam. The final DFT NEXAFS spectrum is the sum of the individual simulated spectra for each target centre in the anion (e.g. each $C$ atom for $\mathrm{CK}$ edge simulations).

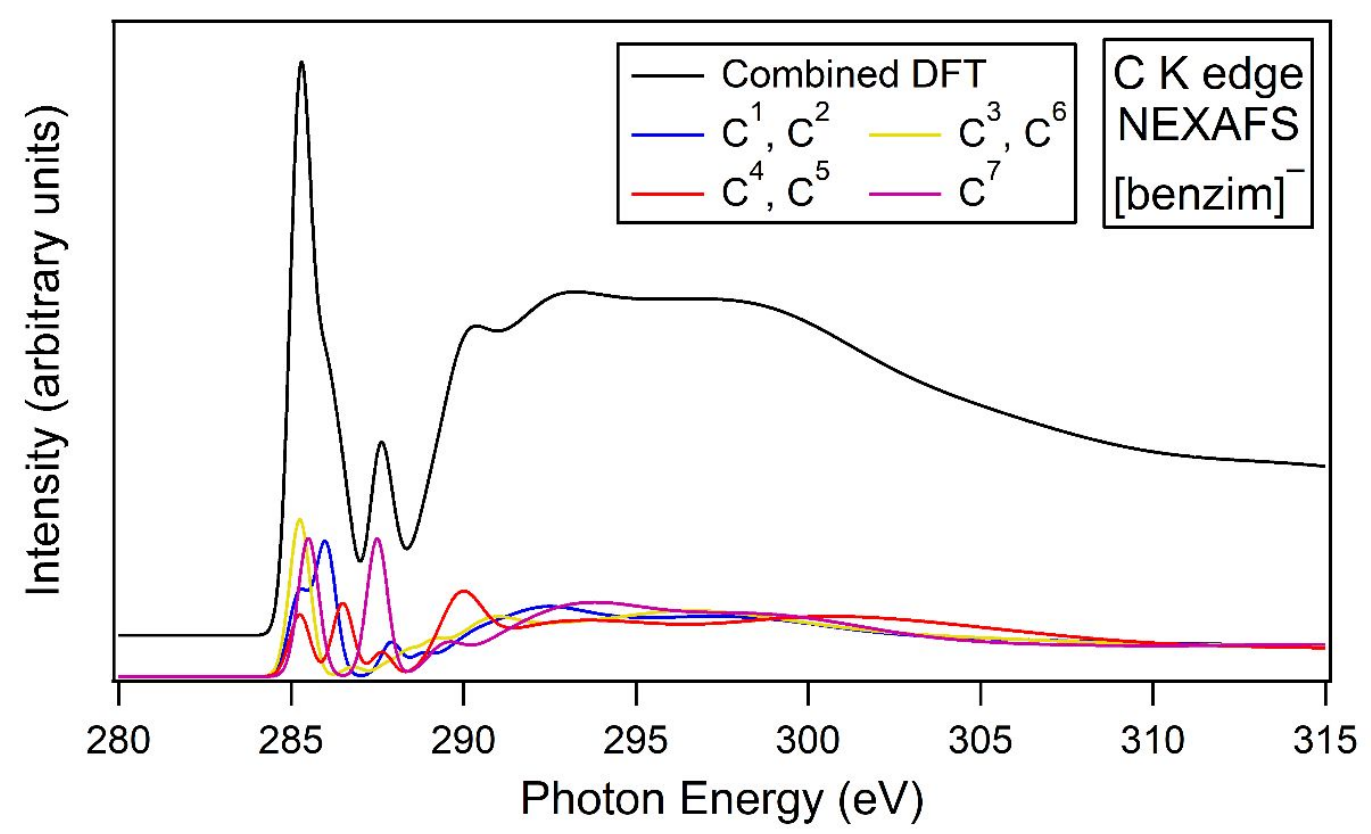

Figure S11. Simulated C K edge NEXAFS spectrum for [P $\left.{ }_{66614}\right][$ benzim]. The final DFT spectrum (black) is the sum of the seven spectra from atoms $C^{1}-C^{7}$.

The simulated C K edge NEXAFS for the IL isolated [benzim]' anion is shown in Figure S11. The breakdown of the combined DFT spectrum (black line) indicates that there are four distinct environments of $C$ atoms in the [benzim] anion: $C^{1,2} ; C^{3,6} ; C^{4,5} ; C^{7}$ (see Figure 1 (a) for assignments). Comparing this to the experimental spectra in Figure 3 , the $\pi^{*}$ peak at 285.3 $\mathrm{eV}$ in the experimental spectrum is largely attributed to transitions from atoms $\mathrm{C}^{3}, \mathrm{C}^{6}$ and $\mathrm{C}^{7}$, with a smaller contribution from $\mathrm{C}^{1}, \mathrm{C}^{2}, \mathrm{C}^{4}$ and $\mathrm{C}^{5}$. The broadening of the $285.3 \mathrm{eV} \pi^{*}$ peak in the DFT spectrum is due to transitions from atoms $C^{1}, C^{2}, C^{4}$ and $C^{5}$ in the [benzim] anion. The $\pi^{*}$ peak at $287.9 \mathrm{eV}$ in the experimental spectrum is largely attributed to transitions from atom $\mathrm{C}^{7}$. This peak occurs at higher photon energies because it is located between the two electronegative $\mathrm{N}$ atoms, consistent with reports for imidazolium-based ILs by Ehlert et al. ${ }^{11}$ 
Although in their case the splitting of the $\pi^{*}$ peak is much narrower, with a split of $0.3 \mathrm{eV}$, compared to a wider separation of $2.6 \mathrm{eV}$ here for [benzim]. The $\pi^{*}$ peak at $290.2 \mathrm{eV}$ is largely due to transitions from atoms $\mathrm{C}^{4}$ and $\mathrm{C}^{5}$, which also have neighbouring $\mathrm{N}$ atoms.

Figure $\mathrm{S} 12$ shows [benzim] ${ }^{-}+\mathrm{CO}_{2}$ over the $\mathrm{C} \mathrm{K}$ edge. The combined DFT spectrum is the sum of all $\mathrm{C}$ atoms in the [benzim] $]^{-}$anion $\left(\mathrm{C}^{1-7}\right)$ and the $\mathrm{C}$ atom in the carbamate species $\left(\mathrm{C}^{8}\right)$. When reacted with $\mathrm{CO}_{2}$, the four environments of $\mathrm{C}$ atoms in [benzim] ' expand into eight: $\mathrm{C}^{1-7}$ for each $\mathrm{C}$ atom in the [benzim] ${ }^{-}$anion, and $\mathrm{C}^{8}$ in the carbamate species. The pre-edge peaks at approximately 282 and $284 \mathrm{eV}$ are attributed to atom $\mathrm{C}^{4}$ near the carbamate. We believe there may be protonated carbamate groups present in the sample so we have also calculated NEXAFS spectra for IL $+\mathrm{COOH}$. The pre-edge features are not present in the protonated carbamate calculation (Figure S13), so they are most likely a result of the interaction between $\mathrm{C}^{4}$ and the COO- moiety. The lower-energy $\pi^{*}$ peak at $285.3 \mathrm{eV}$ in the experimental spectrum is mostly attributed to transitions from atoms $C^{1}, C^{2}, C^{3}$ and $C^{6}$, those furthest from the azole side of the [benzim] ${ }^{-}$anion. Both the broadening of this peak towards higher photon energies and the $\pi^{*}$ peak at $287.2 \mathrm{eV}$ can be largely attributed to the remaining atoms nearer the carbamate: $\mathrm{C}^{4}, \mathrm{C}^{5}$ and $\mathrm{C}^{7}$ (with smaller contributions from $\mathrm{C}^{1}$ and $\mathrm{C}^{2}$ ). We also see that $\mathrm{C}^{8}$ in the carbamate moiety does not contribute to any of the three $\pi^{*}$ peaks or the $\sigma^{*}$ peak at $291.1 \mathrm{eV}$, but rather contributes solely to $\sigma^{*}$ resonances greater than $295 \mathrm{eV}$. This may be because the negative charge was assigned to the singly bonded $O$ atom in the carbamate group (in the software used the negative charge has to be assigned to one atom rather than delocalised).

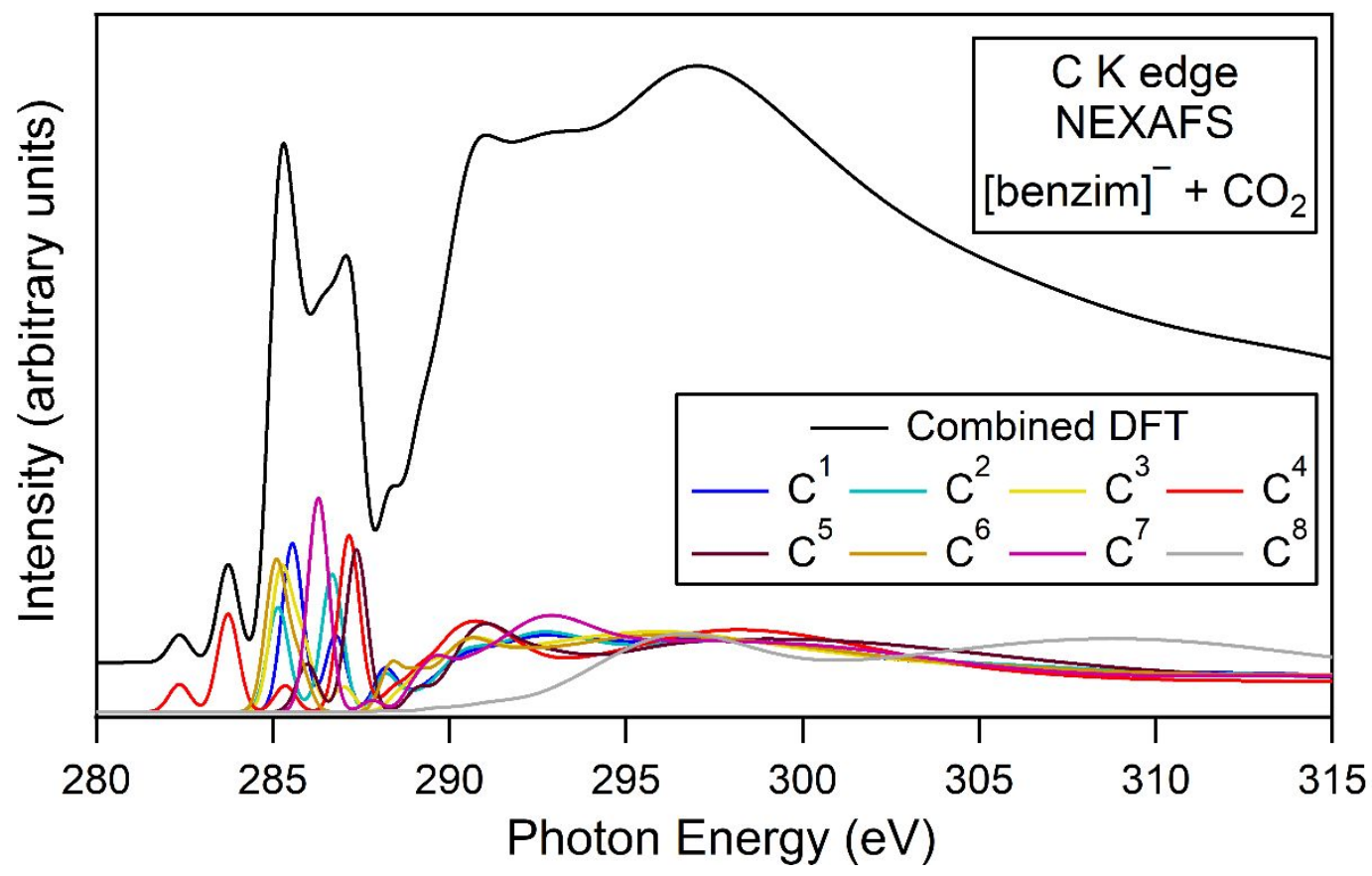

Figure S12. Simulated $C K$ edge NEXAFS spectrum for $\left[P_{66614}\right]\left[\right.$ benzim] $+\mathrm{CO}_{2}$. The final DFT spectrum (black) is the sum of the eight spectra from atoms $C^{1}-C^{8}$. 


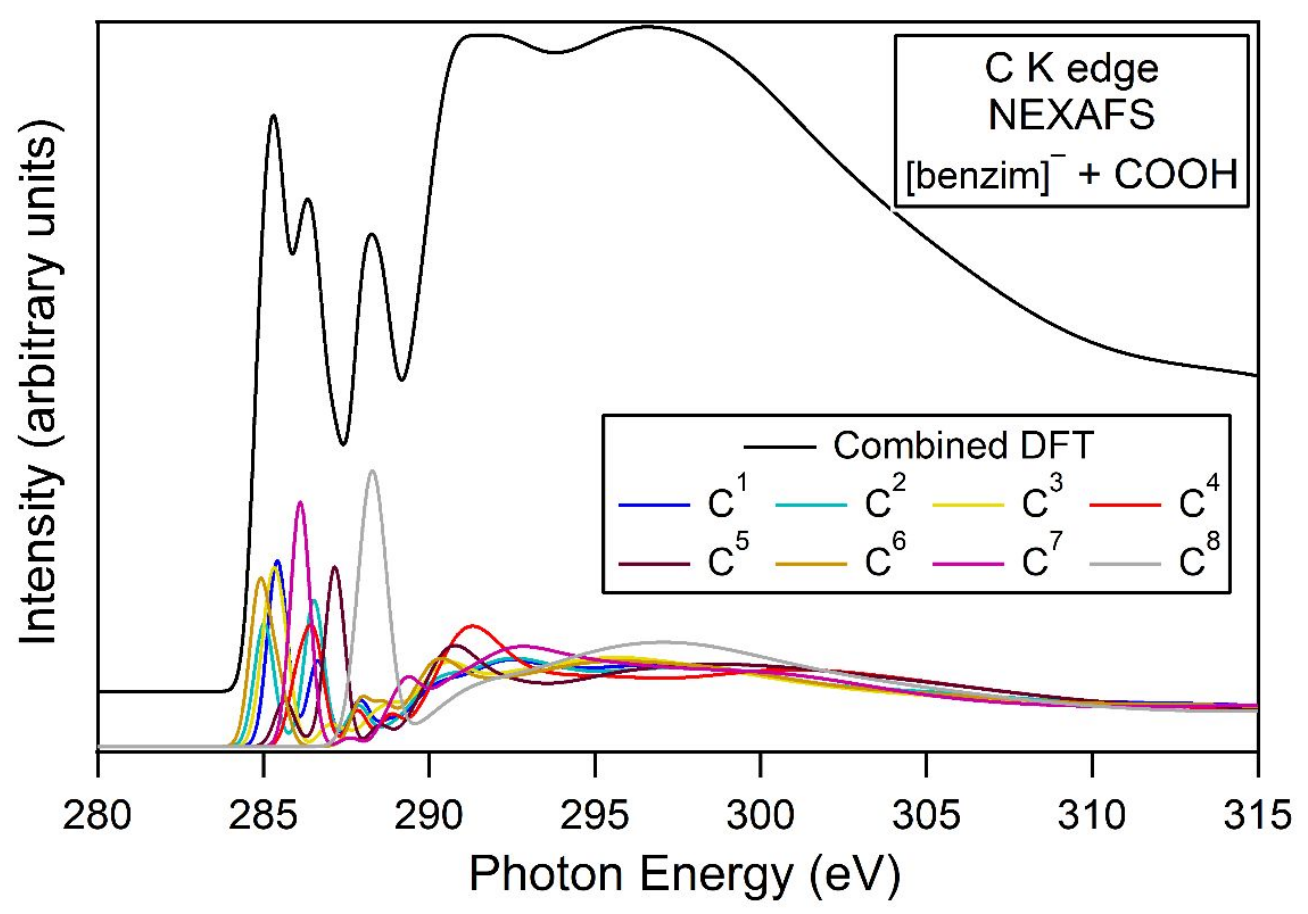

Figure S13: Simulated $C K$ edge NEXAFS spectrum for [ $\left.P_{66614}\right]$ [benzim] $+\mathrm{COOH}$. The final DFT spectrum (black) is the sum of the eight spectra from atoms $C^{1}-C^{8}$.

DFT simulations were also carried out for [benzim] ' over the N K edge as shown in Figure S14. In this case, atoms $\mathrm{N}^{1}$ and $\mathrm{N}^{2}$ have the same spectra, contributing to the final DFT spectrum equally. This is because an isolated [benzim] anion is simulated (in which $\mathrm{N}^{1}$ and $\mathrm{N}^{2}$ are chemically equivalent) and the cation's influence on the anion is not considered. Mercy et al. have shown that the interaction between a $\left[\mathrm{P}_{3333}\right]^{+}$cation and a $[124 \mathrm{Triz}]^{-}$anion results in preferential reactivity at $\mathrm{N}^{1}$, before which the reaction enthalpy was equivalent at both $\mathrm{N}$ sites. ${ }^{12}$ Although it is possible that the influence of the $\left[\mathrm{P}_{66614}\right]^{+}$cation may affect the reaction enthalpy and/or chemical bonding of the $\mathrm{N}$ atoms, it is not expected to be significant. 


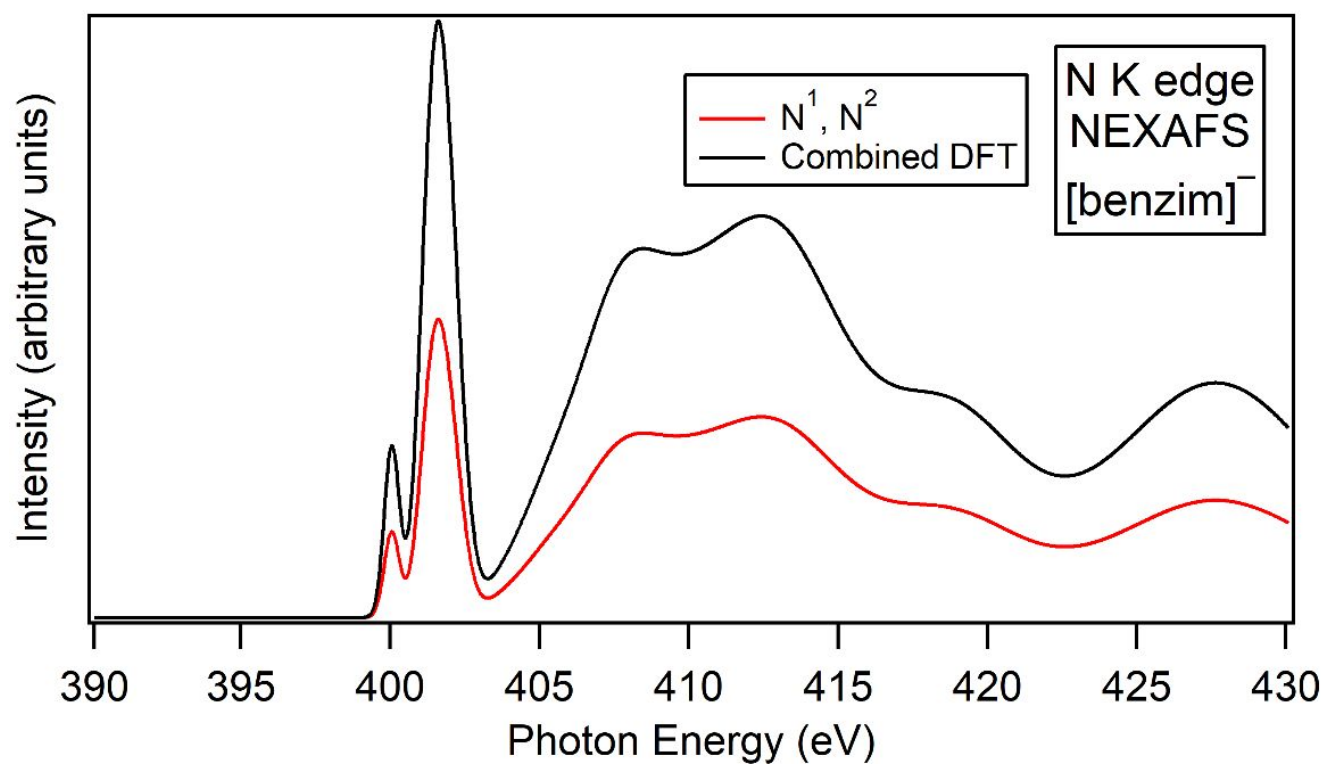

Figure S14. Simulated N K edge NEXAFS spectrum for [ $\left.P_{66614}\right]$ [benzim]. The final DFT spectrum (black) is the sum of the two spectra from atoms $N^{1}$ and $N^{2}$ (red).

When the IL absorbs $\mathrm{CO}_{2}$ (Figure S15) we see that the spectra for $\mathrm{N}^{1}$ and $\mathrm{N}^{2}$ are no longer equivalent due to the formation of carbamate at $\mathrm{N}^{1, \mathrm{r}}$. The formation of this carbamate species destroys the $\mathrm{N}^{1} \rightarrow \pi^{*}$ resonances. Therefore, in the final DFT spectrum, we see that the $\pi^{*}$ peak is solely attributed to transitions from $\mathrm{N}^{2, \mathrm{r}}$ ( $\mathrm{N}^{2}$ in the $\mathrm{CO}_{2}$-reacted anion). The spectra for $\mathrm{N}^{2}$ before exposure (Figure $\mathrm{S} 14$ ) and upon exposure to $\mathrm{CO}_{2}$ (Figure $\mathrm{S} 15, \mathrm{~N}^{2, \mathrm{r}}$ ) are also different, showing that the formation of carbamate at $\mathrm{N}^{1, \mathrm{r}}$ influences $\pi^{*}$ transitions from $\mathrm{N}^{2, r}$.

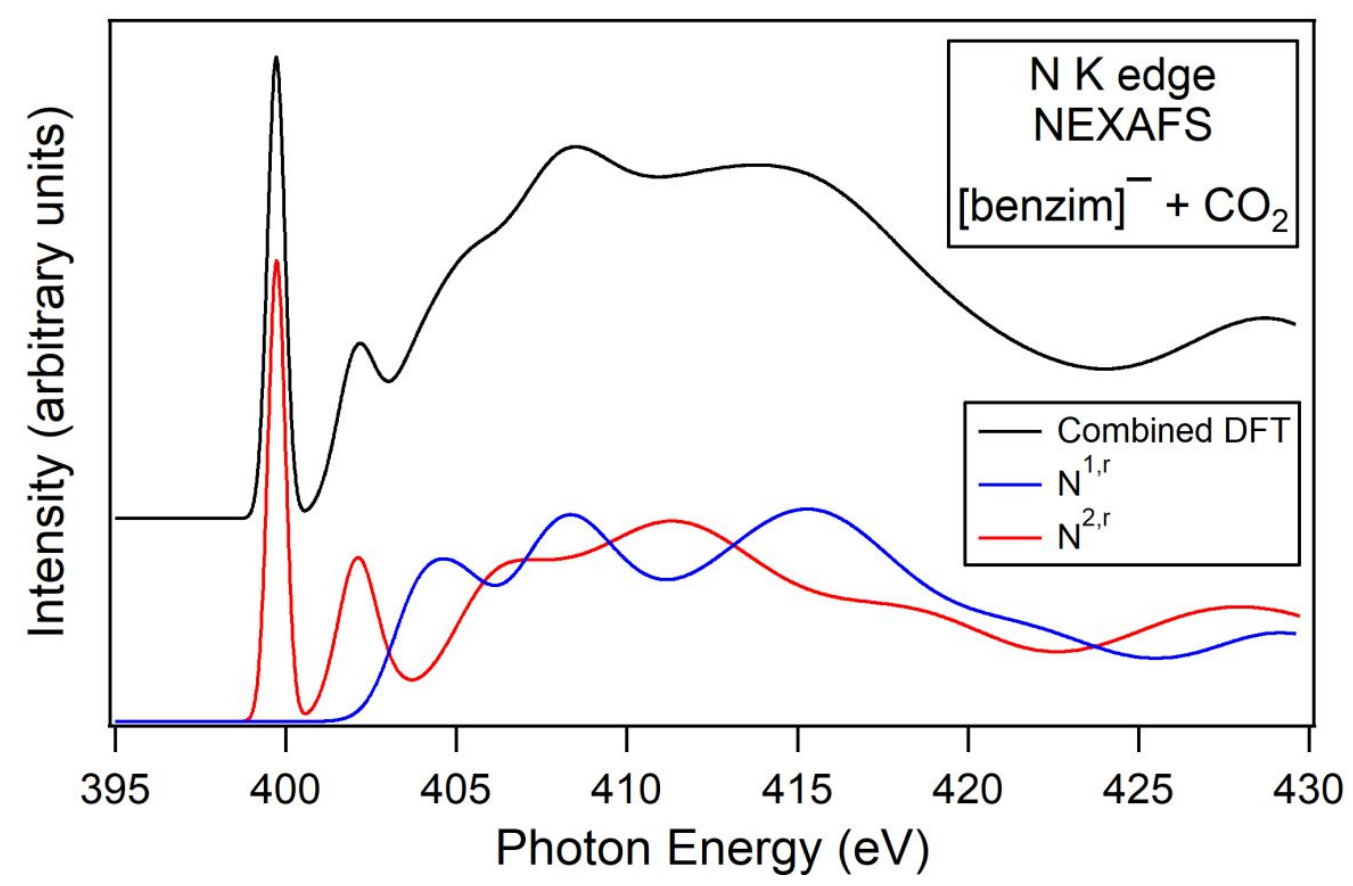

Figure S15. Simulated N K edge NEXAFS spectrum for $\left[P_{66614}\right][$ benzim $]+\mathrm{CO}_{2}$. The final DFT spectrum (black) is the sum of the two spectra from atoms $N^{1, r}$ (red) and $N^{2, r}$ (blue). 
Theoretical DFT spectra were compared to experimental spectra over the $\mathrm{N} \mathrm{K}$ edge for the IL as presented and when exposed to $\mathrm{CO}_{2}$, as shown in Figure S16. For the IL as presented, the lower- and higher-energy $\pi^{*}$ peaks appear at photon energies of 399.9 and $401.4 \mathrm{eV}$, respectively, similar to those in the experimental spectra in Figure S10 (399.9 and $401.7 \mathrm{eV}$ ). The $\sigma^{*}$ peak of the simulated NEXAFS spectrum occurs at the same energy as that of the experimental spectrum $(408.0 \mathrm{eV})$. Thomason et al. studied aqueous imidazole using experimental and theoretical NEXAFS over the N K edge and found a split $\pi^{*}$ feature with two peaks at 400.2 and $401.9 \mathrm{eV} .{ }^{13}$ This is similar to the $\pi^{*}$ peaks of the experimental and simulated NEXAFS of our benzimidazolide-based IL in Figures S10 and S16, respectively. Thomason et al. suggest that the lower- and higher-energy $\pi^{*}$ peaks are assigned to transitions from atoms $\mathrm{N}^{2}$ and $\mathrm{N}^{1}$ in the imidazole, respectively. However, in our case, $\mathrm{N}^{1}$ and $\mathrm{N}^{2}$ are found to contribute in equal parts to the simulated NEXAFS spectrum, as shown in Figure S14. The difference in results may be due to a number of reasons. The main cause of disparity may be due to differences in the chemical environment of the $\mathrm{N}$ atoms ( $\mathrm{N}$ atoms are chemically equivalent in benzimidazolide but not in imidazole) and charge of the two systems.

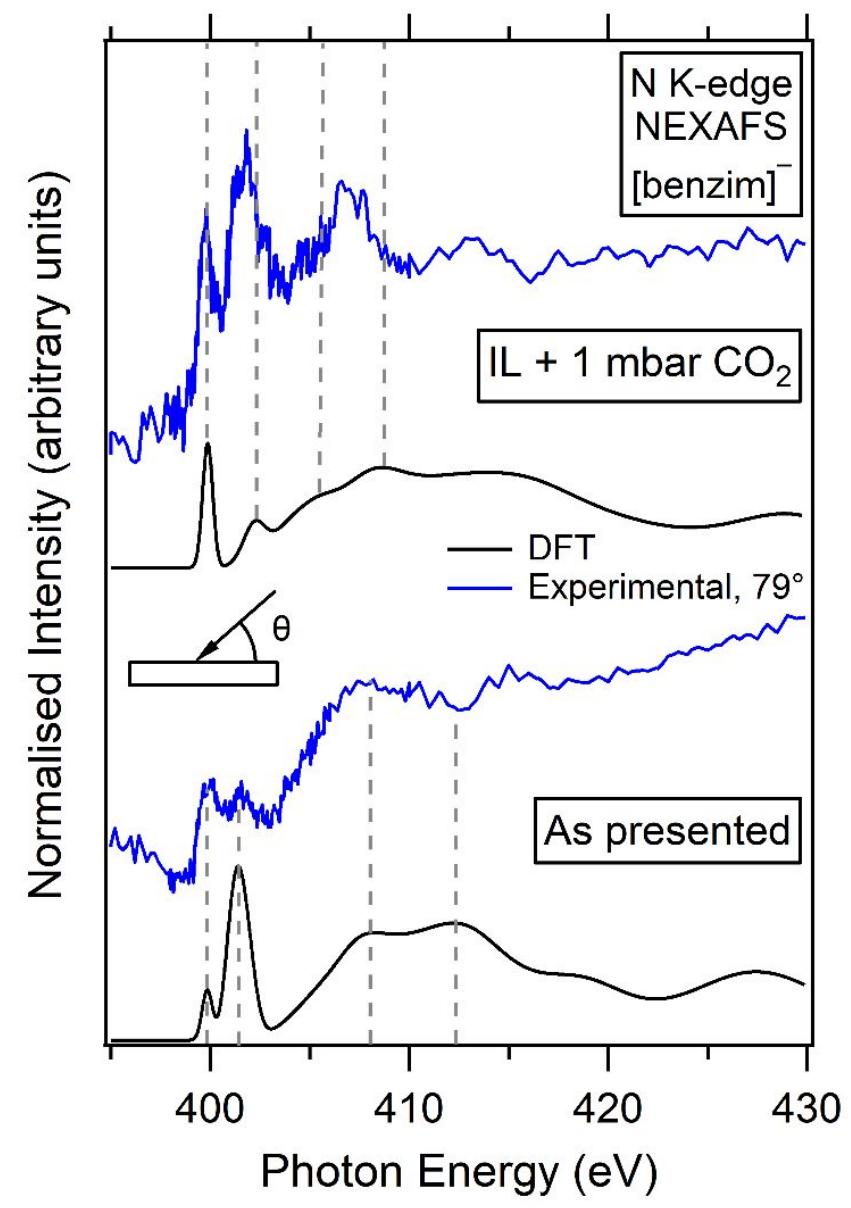

Figure S16. Experimental and simulated (DFT) N K edge NEXAFS of [ $\left.P_{66614}\right]$ [benzim] as presented and when exposed to $1 \mathrm{mbar}$ of $\mathrm{CO}_{2}$. The grey dashed lines highlight features of the DFT spectra for comparison with the experimental spectra. The experimental spectra were taken $79^{\circ}$ from the IL surface.

The IL $+\mathrm{CO}_{2}$ simulated $\mathrm{N} \mathrm{K}$ edge NEXAFS has a split $\pi *$ feature with peaks at 399.9 and 402.3 $\mathrm{eV}$ (compared to 399.9 and $401.7 \mathrm{eV}$ in the experimental spectra). The split $\pi^{*}$ peak arises from transitions from $\mathrm{N}^{2, \mathrm{r}}$ only, the $\mathrm{N}$ atom that remains unreacted upon $\mathrm{CO}_{2}$ absorption 
(Figure S15). The $\sigma^{*}$ peak occurs at a higher energy in the simulated spectrum (408.7 eV) compared to the experimental spectrum $(407.7 \mathrm{eV})$. The $\sigma^{*}$ peak has a slightly greater contribution from $\mathrm{N}^{1, \mathrm{r}}$, the $\mathrm{N}$ atom bonded to carbamate, compared to $\mathrm{N}^{2, \mathrm{r}}$.

We can compare the theoretical N K edge NEXAFS in Figures S14 and S15 to the unoccupied antibonding orbitals of [benzim] ' shown in Figures $4(\mathrm{~b})$ and $(c)$ in the main text. From the $1 \pi^{*}$ orbital we can infer that there are an equal number of excitations from $\mathrm{N}^{1}$ and $\mathrm{N}^{2}$ into the $1 \pi^{*}$ orbital formed around atoms $\mathrm{N}^{1}, \mathrm{C}^{4}, \mathrm{C}^{5}$ and $\mathrm{N}^{2}$. However, the introduction of $\mathrm{CO}_{2}$ results in an asymmetry of this orbital compared to the same orbital in [benzim] ${ }^{-}$pre-exposure. We can assign the two $\pi^{*}$ peaks at 399.9 and $401.7 \mathrm{eV}$ in the IL as presented experimental spectra as $\mathrm{N}^{1,2} \rightarrow 1 \pi^{*}$ and $\mathrm{N}^{1,2} \rightarrow 2 \pi^{*}$ transitions, respectively. Upon exposure to $\mathrm{CO}_{2}$, the peak at 399.9 $\mathrm{eV}$ is assigned to $\mathrm{N}^{2, \mathrm{r}} \rightarrow 1 \pi^{*}$ transitions, while the peak at $401.7 \mathrm{eV}$ is assigned to $\mathrm{N}^{1, \mathrm{r}}$ and $\mathrm{N}^{2, \mathrm{r}}$ $\rightarrow 2 \pi^{*}$ transitions.

\section{$\underline{\text { Anion Orientation }}$}

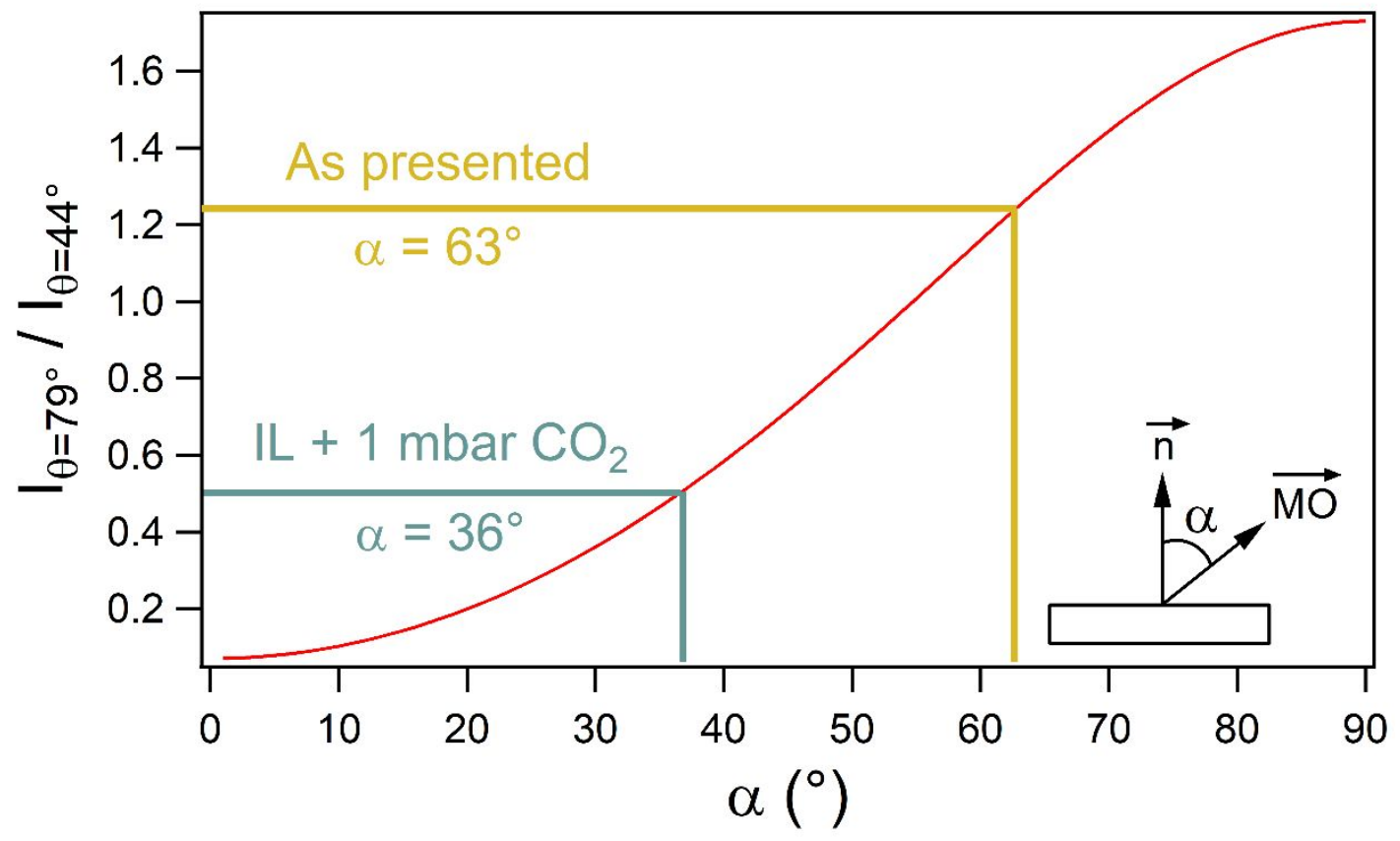

Figure S17: Intensity ratios measured at X-ray angles of incidence $\left(\vartheta=79^{\circ}\right.$ and $\left.\vartheta=44^{\circ}\right)$ calculated as a function of the angle between the surface normal and the molecular orbital, $\alpha$, using the Stöhr equations. Comparing these to the experimental intensity ratios gives $\alpha=63^{\circ}$ for the as presented stage and $\alpha=36^{\circ}$ upon exposure to $1 \mathrm{mbar}$ of $\mathrm{CO}_{2}$.

The angle between the surface normal and the direction of the $\pi^{*}$ molecular orbital is $\alpha$. The ring of the anion is perpendicular to the $\pi^{*}$ molecular orbital, therefore, the tilt of the benzim ring is given by $90^{\circ}-\alpha$. The angle $\alpha$ can be calculated using the Stöhr equations and the intensity ratio of the $\pi^{*}$ resonance peaks at $285 \mathrm{eV}$ in Figure $3 .^{8}$ In the as presented case, the intensities of the $\pi^{*}$ peaks are 0.97 and 0.78 for $\vartheta=79^{\circ}$ and $\vartheta=44^{\circ}$, respectively. This results in a resonance intensity ratio $\left(\mathrm{I}_{\vartheta=79^{\circ}} / \mathrm{I}_{\vartheta=44^{\circ}}\right)$ of 1.24 . When exposed to $1 \mathrm{mbar}$ of $\mathrm{CO}_{2}$, these intensities are 0.59 and 1.19 , respectively, resulting in an intensity ratio of 0.50 . Comparing 
these to the theoretical intensity ratio calculated as a function of $\alpha$ using the Stöhr equations (Figure S17), we can estimate the tilt of the benzim ring to be $27^{\circ}$ from the surface normal in the as presented case. Upon exposure to $\mathrm{CO}_{2}$, the benzim ring reorientates to $54^{\circ}$ from the surface normal. We assume a random azimuthal orientation of the anion and a value of $P=$ 0.85 for the polarisation factor.

\section{References}

(1) Syres, K. L.; Thomas, A. G.; Flavell, W. R.; Spencer, B. F.; Bondino, F.; Malvestuto, M.; Preobrajenski, A.; Grätzel, M. Adsorbate-Induced Modification of Surface Electronic Structure: Pyrocatechol Adsorption on the Anatase $\mathrm{TiO}_{2}(101)$ and Rutile $\mathrm{TiO}_{2}$ (110) Surfaces. J. Phys. Chem. C 2012, 116, 23515-23525.

(2) Taneichi, D.; Haneda, R.; Aramaki, K. A Novel Modification of an Alkanethiol SelfAssembled Monolayer with Alkylisocyanates to Prepare Protective Films against Copper Corrosion. Corros. Sci. 2001, 43, 1589-1600.

(3) Ferraria, A. M.; Boufi, S.; Battaglini, N.; Botelho do Rego, A. M.; Reivilar, M. Hybrid Systems of Silver Nanoparticles Generated on Cellulose Surfaces. Langmuir 2010, 26, 19962001.

(4) Stevens, J. S.; De Luca, A. C.; Pelendritis, M.; Terenghi, G.; Downes, S.; Schroeder, S. L. M. Quantitative Analysis of Complex Amino Acids and RGD Peptides by X-Ray Photoelectron Spectroscopy (XPS). Surf. Interface Anal. 2013, 45, 1238-1246.

(5) Henderson, Z.; Thomas, A. G.; Wagstaffe, M.; Taylor, S. F. R.; Hardacre, C.; Syres, K. L. Reversible Reaction of $\mathrm{CO}_{2}$ with Superbasic Ionic Liquid $\left[\mathrm{P}_{66614}\right]$ [Benzim] Studied with in Situ Photoelectron Spectroscopy. J. Phys. Chem. C 2019, 123, 7134-7141.

(6) Wagstaffe, M.; Jackman, M. J.; Syres, K. L.; Generalov, A.; Thomas, A. G. Ionic Liquid Ordering at an Oxide Surface. ChemPhysChem 2016, 17, 3430-3434.

(7) Cremer, T.; Stark, M.; Deyko, A.; Steinrück, H. P.; Maier, F. Liquid/Solid Interface of Ultrathin lonic Liquid Films: $\left[\mathrm{C}_{1} \mathrm{C}_{1} I \mathrm{~m}\right]\left[\mathrm{Tf}_{2} \mathrm{~N}\right]$ and $\left[\mathrm{C}_{8} \mathrm{C}_{1} \mathrm{Im}\right]\left[\mathrm{Tf} \mathrm{f}_{2} \mathrm{~N}\right]$ on $\mathrm{Au}(111)$. Langmuir 2011, 27, 3662-3671.

(8) Stöhr, J. NEXAFS Spectroscopy; Springer: Berlin, 2003.

(9) Walsh, J. F.; Dhariwal, H. S.; Gutiérrez-Sosa, A.; Finetti, P.; Muryn, C. A.; Brookes, N. B.; Oldman, R. J.; Thornton, G. Probing Molecular Orientation in Corrosion Inhibition via a NEXAFS Study of Benzotriazole and Related Molecules on Cu(100). Surf. Sci. 1998, 415, 423-432.

(10) Grillo, F.; Garrido Torres, J. A.; Treanor, M. J.; Larrea, C. R.; Götze, J. P.; Lacovig, P.; Früchtl, H. A.; Schaub, R.; Richardson, N. V. Two-Dimensional Self-Assembly of Benzotriazole on an Inert Substrate. Nanoscale 2016, 8, 9167-9177. 
(11) Ehlert, C.; Holzweber, M.; Lippitz, A.; Unger, W. E. S.; Saalfrank, P. A Detailed Assignment of NEXAFS Resonances of Imidazolium Based Ionic Liquids. Phys. Chem. Chem. Phys. 2016, 18, 8654-8661.

(12) Mercy, M.; Rebecca Taylor, S. F.; Jacquemin, J.; Hardacre, C.; Bell, R. G.; De Leeuw, N. $\mathrm{H}$. The Addition of $\mathrm{CO}_{2}$ to Four Superbase Ionic Liquids: A DFT Study. Phys. Chem. Chem. Phys. 2015, 17, 28674-28682.

(13) Thomason, M. J.; Seabourne, C. R.; Sattelle, B. M.; Hembury, G. A.; Stevens, J. S.; Scott, A. J.; Aziz, E. F.; Schroeder, S. L. M. Self-Association of Organic Solutes in Solution: A NEXAFS Study of Aqueous Imidazole. Faraday Discuss. 2015, 179, 269-289. 